\title{
ERRATA AND ADDENDA: PRE-TANGO STRUCTURES ON CURVES
}

(Tohoku Math. J. 54 (2002), 227-237)

\author{
Yoshifumi TAKEDA AND KÔJI YOKOGAWA
}

(Received May 29, 2003, revised July 16, 2003)

The purpose of the present note is to correct errors in the authors' article [1] and to add a supplement.

To begin with, we correct typographical errors. The equation " $x^{p}=z_{i}\left(1+a_{i} z_{i}^{n}\right)^{\beta}$ " in the fifth and sixth lines from the bottom of p. 231 should read " $x_{i}^{p}=z_{i}\left(1+a_{i} z_{i}^{n}\right)^{\beta}$ ".

In addition, we correct a mathematical error in the statement of Theorem 3.2, which arises from an incorrect estimate of the degree of the torsion sheaf $\mathcal{O}_{\tilde{C}} / \mathcal{O}_{\hat{C}}$ in the twelfth line from the bottom of p. 236. The correct extimate is $\operatorname{deg} \mathcal{O}_{\tilde{C}} / \mathcal{O}_{\hat{C}}<(i-1+p)(p-1)$. Moreover, we have another error in the proof, namely, the equation $y^{2}=x^{d}-x$ in the third line from the bottom of p. 236. Indeed, the polynomial $x^{d}-x$ has multiple roots in the case of $d \equiv 1(\bmod p)$. Then the equation does not define any hyperelliptic curve. In order to correct these errors, the following modifications are necessary.

First, we replace the statement and the proof of Theorem 2.4 on p. 233 with the following

THEOREM 2.4. Retain the same notation and assumptions as above. Suppose that we have a non-negative integerl and a pre-Tango structure $\mathcal{L}^{n} \hookrightarrow \mathcal{B}$ on $C$ such that $n>p^{2}+$ lp . Then $Z$ has non-closed global differential 1-forms if $H^{0}\left(C, \mathcal{L}^{(l+1) p}\right) \underset{\neq}{\subsetneq} H^{0}\left(C, \mathcal{L}^{(l+1) p} \otimes \mathcal{O}_{C}\right.$ $\left.\Phi_{*} \mathcal{O}_{\Sigma}\right)$.

PROOF. Since $Y$ is defined locally by $v_{i}^{p}=u_{i}^{n}+a_{i}$, we have $-n u_{i}^{n-1} \mathrm{~d} u_{i}=\mathrm{d} a_{i}$ by exterior differentiation. Let $\mathcal{P}$ be the $\mathcal{O}_{C}$-submodule of $\Omega_{C / k}$ locally generated by $\mathrm{d} a_{i}$, where $\Omega_{C / k}$ is the sheaf of differentials on $C$. We then obtain an injection $\Phi^{*} \mathcal{P} \otimes \mathcal{O}_{Z} \mathcal{O}_{Z}((n-$ 1) $\Sigma) \rightarrow \Omega_{Z / k}^{1}$, where $\Omega_{Z / k}^{1}$ is the sheaf of differentials on $Z$. We consider the global differential 1-forms in $\Phi^{*} \mathcal{P} \otimes \mathcal{O}_{Z} \mathcal{O}_{Z}((n-1) \Sigma)$. Over $U_{i}$, we have

$$
\begin{aligned}
u_{i}^{l} \mathrm{~d} u_{i} & =-\frac{1}{n} z_{i}^{n-1-l} \mathrm{~d} a_{i}, \\
v_{i} u_{i}^{l} \mathrm{~d} u_{i}= & -\frac{1}{n} y_{i} z_{i}^{m(p-1)-r-1-l} \mathrm{~d} a_{i}, \\
& \vdots \\
v_{i}^{p-1} u_{i}^{l} \mathrm{~d} u_{i} & =-\frac{1}{n} y_{i}^{l} z_{i}^{m-r-1-l} \mathrm{~d} a_{i} .
\end{aligned}
$$

The left hand side of each equation shows the differential form is regular on $Y$, while the right hand side shows that the differential form is regular on $X$ when $m-r-1-l>0$. Since $n>p^{2}+l p$, we have $m>r+1+l$. Therefore, it follows that $u_{i}^{l} \mathrm{~d} u_{i}, v_{i} u_{i}^{l} \mathrm{~d} u_{i}, \ldots, v_{i}^{p-1} u_{i}^{l} \mathrm{~d} u_{i}$ 
are sections in $\Phi^{*} \mathcal{P} \otimes \mathcal{O}_{Z}((n-1) \Sigma)\left(\Phi^{-1}\left(U_{i}\right)\right)$ and so in $\mathcal{P} \otimes \Phi_{*} \mathcal{O}_{Z}((n-1) \Sigma)\left(U_{i}\right)$. Let $\mathcal{Q}$ be the $\mathcal{O}_{C}$-submodule of $\mathcal{P} \otimes_{\mathcal{O}_{C}} \Phi_{*} \mathcal{O}_{Z}((n-1) \Sigma)$ generated locally by $u_{i}^{l} \mathrm{~d} u_{i}$. Then $\mathcal{Q}$ is isomorphic to $\mathcal{L}^{(l+1) p}$. Consider the direct image of the structure sheaf $\mathcal{O}_{\Sigma}$ of $\Sigma$ by $\Phi$. Since $\Phi_{*} \mathcal{O}_{\Sigma}$ is generated locally by $1, v_{i}, \ldots, v_{i}^{p-1}$ as an $\mathcal{O}_{C}$-module, we have an inclusion $\mathcal{Q} \otimes \mathcal{O}_{C} \Phi_{*} \mathcal{O}_{\Sigma} \subset \mathcal{P} \otimes \mathcal{O}_{C} \Phi_{*} \mathcal{O}_{Z}((n-1) \Sigma)$. We summarize these inclusions of $\mathcal{O}_{C}$-modules as follows:

$$
\mathcal{Q} \subset \mathcal{Q} \otimes_{\mathcal{O}_{C}} \Phi_{*} \mathcal{O}_{\Sigma} \subset \mathcal{P} \otimes_{\mathcal{O}_{C}} \Phi_{*} \mathcal{O}_{Z}((n-1) \Sigma) \subset \Phi_{*} \Omega_{Z / k}^{1}
$$

Since $\mathcal{Q}$ is locally generated by $u_{i}^{l} \mathrm{~d} u_{i}$, any section of $\mathcal{Q}$ is a closed differential 1 -form on $Z$. On the other hand, $\mathcal{Q} \otimes \mathcal{O}_{C} \Phi_{*} \mathcal{O}_{\Sigma}$ is locally generated by $u_{i}^{l} \mathrm{~d} u_{i}, v_{i} u_{i}^{l} \mathrm{~d} u_{i}, \ldots, v_{i}^{p-1} u_{i}^{l} \mathrm{~d} u_{i}$. So we conclude that the sections of $\mathcal{Q} \otimes \mathcal{O}_{C} \Phi_{*} \mathcal{O}_{\Sigma}$ which are not in $\mathcal{Q}$ are non-closed differential 1 -forms on $Z$. Hence we arrive at the required assertion.

Thereby, the condition $(*)$ on p. 235 should be replaced by

The degree $n$ is relatively prime to $p$ and greater than $p^{2}+3 p$, and

$$
H^{0}\left(C, \mathcal{L}^{4 p}\right) \underset{\neq}{\subset} H^{0}\left(C, \mathcal{L}^{4 p} \otimes_{\mathcal{O}_{C}} \hat{F}_{*} \mathcal{O}_{\hat{C}}\right) .
$$

Needless to say, this condition arises from the assumption of Theorem 2.4, corrected above, with $l=3$.

Next, we correct Theorem 3.2 and its proof on p. 236. Here we note that the corrected statement of Theorem 3.2 includes the case of characteristic $p=3,5,7$. The first and the third paragraphs of the new proof proceed almost the same lines as the original ones. We shall, however, restate them for reader's convenience.

THEOREM 3.2. If $2 g \geq p^{3}+3 p^{2}+4 p$ and $p \geq 3$, then the locus $\mathcal{T}_{g}$ contains $a$ variety of dimension $g-1$.

Proof. Consider the subvariety $\mathcal{H}_{g}$ of $\mathcal{M}_{g}$ consisting of hyperelliptic curves of genus $g$. It is well-known that the dimension of $\mathcal{H}_{g}$ is $2 g-1$. Since each curve in $\mathcal{H}_{g}$ is given by an equation of type $y^{2}=x(x-1)\left(x-a_{1}\right) \cdots\left(x-a_{2 g-1}\right)$, we have a rational mapping $A^{2 g-1} \rightarrow \mathcal{H}_{g}$ whose image coincides with $\mathcal{H}_{g}$. Let $C$ be a curve in $\mathcal{H}_{g}$ defined by the equation $y^{2}=x(x-1)\left(x-a_{1}\right) \cdots\left(x-a_{2 g-1}\right)$, and let

$$
\left(\begin{array}{ccc}
c_{11} & \ldots & c_{1 g} \\
\vdots & & \vdots \\
c_{g 1} & \ldots & c_{g g}
\end{array}\right)
$$

be the Hasse-Witt matrix of $C$, that is, the matrix representing the mapping on $H^{0}\left(C, \Omega_{C}\right)$ induced from the Cartier operator $\mathcal{C}$, with respect to the basis $\left\{\mathrm{d} x / y, x \mathrm{~d} x / y, \ldots, x^{g-1} \mathrm{~d} x / y\right\}$. Note that all entries of this matrix are polynomials in $a_{1}, \ldots, a_{2 g-1}$. For $1 \leq i \leq g$, consider the subvariety $S_{i}$ of $\boldsymbol{A}^{2 g-1}$ defined by $c_{i 1}=\cdots=c_{i g}=0$. Namely, $S_{i}$ is the subvariety consisting of those points corresponding to the curves such that all entries in the $i$-th columns of their Hasse-Witt matrices are zero. Let $V_{i}$ be the image in $\mathcal{H}_{g}$ of $S_{i}$. If $V_{i}$ is not empty, then we have $\operatorname{dim} V_{i} \geq g-1$. 
Suppose that $V_{i}$ is not empty with $i \leq p$. Let $C$ be a hyperelliptic curve corresponding to a point in $V_{i}$. Then we have $\mathcal{C}\left(x^{i-1} \mathrm{~d} x / y\right)=0$. Since $i \leq p$ and $2 g \geq p^{3}+3 p^{2}+4 p$, we have $2 g-2-2(i-1)-2 p \geq p^{3}+3 p^{2}$. By virtue of the previous lemma, we have a pre-Tango structure $\mathcal{L}^{n}$ with $\mathcal{L}=\mathcal{O}_{C}\left(P_{\infty}\right)$ and $n>p^{2}+3 p$. Consider the exact sequence

$$
0 \rightarrow \mathcal{O}_{\hat{C}} \rightarrow \mathcal{O}_{\tilde{C}} \rightarrow \mathcal{O}_{\tilde{C}} / \mathcal{O}_{\hat{C}} \rightarrow 0
$$

on $C$, where $\tilde{C}$ is the normalization of $\hat{C}$ (cf. Remark 2.7). Since the structure sheaf of $\hat{C}$ is locally generated by $1, q_{i}, \ldots, q_{i}^{p-1}$ as $\mathcal{O}_{C}$-module, we have a filtration

$$
\mathcal{O}_{C} \subset \mathcal{E}_{1} \subset \cdots \subset \mathcal{E}_{p-1}=\mathcal{O}_{\hat{C}}
$$

where $\mathcal{E}_{\lambda}$ is locally generated by $1, q_{i}, \ldots, q_{i}^{\lambda}$ as $\mathcal{O}_{C}$-module. Then each successive quotient $\mathcal{E}_{\lambda} / \mathcal{E}_{\lambda-1}$ is isomorphic to $\mathcal{L}^{\lambda n}$. Therefore, we obtain $\chi\left(\mathcal{O}_{\hat{C}}\right)=\chi\left(\mathcal{O}_{C}\right)+\sum_{\lambda=1}^{p-1} \chi\left(\mathcal{L}^{\lambda n}\right)$. It follows from the Riemann-Roch theorem that $\chi\left(\mathcal{O}_{\hat{C}}\right)=p(1-g)+n p(p-1) / 2$. Since $n p>2 g-2-2(i-1)-2 p$, the exact sequence $(6)$ implies that $\mathcal{O}_{\tilde{C}} / \mathcal{O}_{\hat{C}}$ is a torsion sheaf of degree less than $(i-1+p)(p-1)$. By tensoring $\mathcal{L}^{4 p}$ over $\mathcal{O}_{C}$ to $(6)$, we have

$$
0 \rightarrow \mathcal{O}_{\hat{C}} \otimes \mathcal{L}^{4 p} \rightarrow \mathcal{O}_{\tilde{C}} \otimes \mathcal{L}^{4 p} \rightarrow \mathcal{O}_{\tilde{C}} / \mathcal{O}_{\hat{C}} \rightarrow 0
$$

Since $\mathcal{L}=\mathcal{O}_{C}\left(P_{\infty}\right)$, we obtain $\operatorname{dim} H^{0}\left(C, \mathcal{L}^{4 p}\right)=2 p+1$. Moreover, we know that $\mathcal{O}_{\tilde{C}} \otimes$ $\mathcal{L}^{4 p}$ is isomorphic to $\mathcal{L}^{4 p^{2}}$. Therefore, we have $\operatorname{dim} H^{0}\left(\mathcal{O}_{\tilde{C}} \otimes \mathcal{L}^{4 p}\right)=2 p^{2}+1$. Since $\operatorname{deg} \mathcal{O}_{\tilde{C}} / \mathcal{O}_{\hat{C}}<(i-1+p)(p-1) \leq(2 p-1)(p-1)$, we know that $\operatorname{dim} H^{0}\left(\mathcal{O}_{\hat{C}} \otimes \mathcal{L}^{4 p}\right)>3 p$. Hence it follows that $H^{0}\left(C, \mathcal{L}^{4 p}\right) \underset{\neq}{\subsetneq} H^{0}\left(C, \mathcal{L}^{4 p} \otimes \mathcal{O}_{C} \hat{F}_{*} \mathcal{O}_{\hat{C}}\right)$. Therefore, we conclude that $V_{i}$ is contained in $\mathcal{T}_{g}$. Now we have only to show that one of $\left\{V_{i} \mid i \leq p\right\}$ is not empty.

Suppose that $g \not \equiv 0(\bmod p)$. Set $d=2 g+1$ and let $C$ be the hyperelliptic curve determined by $y^{2}=x^{d}-x$. Consider the differential form $\mathrm{d} x / y$ and its image by the Cartier operator. We then have

$$
y \mathcal{C}\left(\frac{\mathrm{d} x}{y}\right)=\mathcal{C}\left(y^{p-1} \mathrm{~d} x\right)=\mathcal{C}\left(\left(x^{d}-x\right)^{(p-1) / 2} \mathrm{~d} x\right) .
$$

Only the terms

$$
x^{(p-1) / 2}, \quad x^{d+(p-1) / 2-1}, \quad \ldots, \quad x^{d((p-1) / 2-1)+1}, \quad x^{d(p-1) / 2}
$$

appear in $\left(x^{d}-x\right)^{(p-1) / 2}$. If $\mathcal{C}(\mathrm{d} x / y)=0$, then $V_{1}$ is not empty. Assume that $\mathcal{C}(\mathrm{d} x / y) \neq 0$. Then there exists an integer $l$ such that $0 \leq l \leq(p-1) / 2$ and $d l+(p-1) / 2-l \equiv p-1$, that is, $l(d-1)+(p-1) / 2 \equiv p-1(\bmod p)$. Note that $d-1 \not \equiv 0(\bmod p)$. Let $j$ be the integer such that $0 \leq j \leq p-1$ and $j \equiv(l+1)(d-1)(\bmod p)$. Consider the differential form $x^{j} \mathrm{~d} x / y$. We then have $j+(p-1) / 2 \equiv(l+1)(d-1)+(p-1) / 2$. Moreover, since the mapping $\boldsymbol{Z} / p \boldsymbol{Z} \rightarrow \boldsymbol{Z} / p \boldsymbol{Z} ; l \mapsto l(d-1)+(p-1) / 2$ is bijective, we know that the integers $j+(p-1) / 2, j+d+(p-1) / 2-1, \ldots, j+d((p-1) / 2-1)+1, j+d(p-1) / 2$ are not equivalent to $p-1$ modulo $p$. So, we have $\mathcal{C}\left(x^{j} \mathrm{~d} x / y\right)=0$. Hence $V_{j+1}$ is not empty.

Suppose that $g \equiv 0(\bmod p)$. Let $C$ be the hyperelliptic curve defined by $y^{2}=x^{2 g+1}+$ $x^{2}-2 x$. Only the terms of degree $(2 g+1) \alpha+2 \beta+\gamma$ appear in $\left(x^{2 g+1}+x^{2}-2 x\right)^{(p-1) / 2}$, where $\alpha, \beta, \gamma$ are non-negative integers satisfying $\alpha+\beta+\gamma=(p-1) / 2$. By a simple computation, 
we see that those degrees are in the form $2 g \alpha+p-(\alpha+\gamma)-1$. Since $g \equiv 0(\bmod p)$ and $0 \leq \alpha+\gamma \leq(p-1) / 2$, it follows that $2 g \alpha+p-(\alpha+\gamma)-1+(p+1) / 2 \not \equiv p-1(\bmod p)$. So, we have $\mathcal{C}\left(x^{(p+1) / 2} \mathrm{~d} x / y\right)=0$. Hence $V_{(p+1) / 2+1}$ is not empty.

We close this note with the following remarks on Theorem 3.2.

REMARK 3.3 (Tsuda[3]). Retain the same notation and assumptions as in Theorem 3.2. By examining accurately the entries of the Hasse-Witt matrix, we can verify that some of the entries are zero. Hence the locus $\mathcal{T}_{g}$ contains a variety of dimension $g+\lceil g / p\rceil-1$, where $\lceil a\rceil$ denotes the smallest integer greater than or equal to $a$.

REMARK 3.4 (Takeda[2]). In the case of characteristic two, on any curve of genus greater than 11, there exists a pre-Tango structure which induces a smooth complete surface having non-closed global differential 1-forms.

Acknowledgment. Some of the errors in the original article are indicated by Miss Noriko Tsuda. The authors would like to express their gratitude for her comments and suggestions.

\section{REFERENCES}

[ 1] Y. TAKeda And K. Yokogawa, Pre-Tango structures over curves, Tohoku Math. J. 54 (2002), 227-237.

[2] Y. TAKEDA, Pre-Tango structures in characteristic two, Japan. J. Math 28 (2002), 81-86.

[ 3 ] N. TsudA, Pre-Tango structures on hyperelliptic curves (Japanese), Master's Thesis.

DEPARTMENT OF MATHEMATICS NARA WOMEN'S UNIVERSITY

NARA 630-8506

JAPAN

\section{DEPARTMENT OF MATHEMATICS}

OCHANOMIZU UNIVERSITY

OTSUKA 2-1-1, BUNKYO-KU

TOKYO 112-0012

JAPAN 